\title{
Drop tower tests of Taiji-1 inertial sensor substitute
}

\author{
Jian Min ${ }^{1}$, Zuo-Lei Wang ${ }^{1}$, Yun-Peng Li ${ }^{1}$, Wen-Ze Tao ${ }^{1}$, Cun-Hui Li $\mathbb{D}^{1}$, Jun-Gang Lei ${ }^{1}$, Dong-Xue Xi $\mathbb{D}^{1 凶}$, Da Fan $^{2}$ and Jun-Biao Wang ${ }^{3}$
}

Taiji-1, which is the first technical verification satellite of China's Space Gravitational Wave Detection Program, was successfully launched on August 31, 2019. The mission aimed to investigate the key technologies used in space gravitational wave detection. The inertial sensor, which was one of the main payloads, measured the residual acceleration of the satellite, and verified the dragfree control technology. Its performance was crucial to the success of the Taiji-1 mission. To ensure its performance in orbit, the inertial sensor was fully evaluated prior to launch. Owing to the gravitational acceleration on the ground, it is impossible to verify all the properties of the inertial sensor in a routine laboratory. A feasible method to conduct such tests is to use a drop tower. To guarantee the safety of the inertial sensor, a substitute was used with similar structure and circuit design. A total of 20 falls in three groups were completed, a set of research methods was established, and the importance of conducting simulations before the drop tests was verified. For the first time, the switch of different circuit gains in a drop tower test has been achieved and the National Microgravity Laboratory of China (NMLC) drop tower's residual accelerations in three dimensions were measured. The results demonstrated that the microgravity level of the drop tower can reach about $58 \mu_{0}$ in the fall direction and $13 \mu g_{0}$ along the horizontal axes.

npj Microgravity (2021)7:25 ; https://doi.org/10.1038/s41526-021-00154-8

\section{INTRODUCTION}

Following the success of ground-based gravitational wave (GW) detection ${ }^{1,2}$, space-based GW detection offers a novel method for studying the universe ${ }^{3-6}$. Compared to LIGO-like GW detectors on the ground, LISA-like GW detectors in space aim for a lower frequency range of $0.1 \mathrm{mHz} 1 \mathrm{~Hz}$. The Taiji program is one of China's space GW exploration programs and is planned to launch around $2033^{7,8}$. An inertial sensor will be used as a position reference for both drag-free control and laser interference technologies. To ensure the success of the program, its accuracy needs to be $5 \sim 10 \mathrm{pm} / \sqrt{\mathrm{Hz}}^{9}$.

The first technical verification satellite of the Taiji program, known as Taiji-1, was launched on August 31, $2019^{10}$. One of the main payloads on Taiji-1 $1^{11-13}$ is the inertial sensor. To estimate its performance, a series of ground tests needs to be conducted. However, due to the gravitational acceleration in the direction of gravity, it is almost impossible to fully evaluate the in-orbit performance of the inertial sensor in a routine laboratory. Furthermore, in order to improve the stability of the control system and achieve higher measurement accuracy, the circuit gains need to be switched at times. However, this cannot be performed on the ground with a high-voltage suspension along the X-axis (Fig. 1) (i.e., the direction of gravity). For the above reasons, it is important to simulate the in-orbit performance with the inertial sensor prior to launch. An appropriate solution is to use a drop tower to simulate a microgravity environment and then conduct systematic tests.

For both the Aster and GOCE accelerometers, the ZARM drop tower was used for acceptance testing ${ }^{14,15}$. For the MICROSCOPE accelerometer, the ZARM drop tower was also used to demonstrate the control capability of the two coaxial test masses ${ }^{16,17}$. In December 2016, researchers conducted a series of drop tower tests with a reduced prototype of MicroSTAR at ZARM to verify the concept and optimize the accelerometer control loop ${ }^{18}$.
For the experiments presented in this paper, the drop tower of the National Microgravity Laboratory of China (NMLC) in Beijing was used, which is capable of providing a free-fall time of about $3.5 \mathrm{~s}$ and a microgravity level of about $10^{-5} \mathrm{~g}_{0}$, where $\mathrm{g}_{0}$ is the local gravitational acceleration ${ }^{19}$. Two groups of mode-switch experiments were performed and three different control modes were used. These experiments allowed not only the performance evaluation of the inertial sensor, but also demonstrated the effect of circuit gain switching on the control loop under microgravity environment.

To ensure the safety of the real inertial sensor, a substitute inertial sensor (SIS) was used in the drop tower test. The SIS had almost the same structure with the actual inertial sensor, the only difference being the vacuum device on the top of the sensorhead. The SIS had a vacuum chamber to maintain a vacuum on the ground (Fig. 1a), while in the in-orbit sensor, this chamber was removed and the vacuum in space was used (Fig. 1b). Moreover, the SIS had the same circuit design as that of the inertial sensor on Taiji-1, and in order to adapt to the microgravity level of the drop tower, the gain of the amplifier circuit was adjusted. The electrode housing and the axial diagram of the sensor-head are illustrated in Fig. 1c. There were three electrode pairs along the $X$-axis, two along the Z-axis, and one along the Y-axis.

A cuboid-shaped gold-plated titanium alloy was used as the test mass to perceive the acceleration of the sensor. The test mass was connected to the measure and control unit (MCU) through a gold wire to provide it with a DC bias voltage $\left(V_{p}\right)$ and a position detection AC signal $\left(V_{\mathrm{d}}\right)$. The electrode housing surrounding the test mass was made of gold-plated microcrystalline glass, taking into consideration the thermal stability and magnetic susceptibility of the sensor. With the vacuum sealing structure, the vacuum pressure of the SIS could be maintained below $10^{-5} \mathrm{~Pa}$ on the ground.

\footnotetext{
${ }^{1}$ National Key Laboratory of Science and Technology on Vacuum Technology and Physics, Lanzhou Institute of Space Technology and Physics, Lanzhou, China. ${ }^{2}$ Qian Xuesen Laboratory of Space Technology, Beijing, China. ${ }^{3}$ National Microgravity Laboratory, Institute of Mechanics, Chinese Academy of Sciences, Beijing, China. ${ }^{凶}$ email: nihong621@126. com
} 

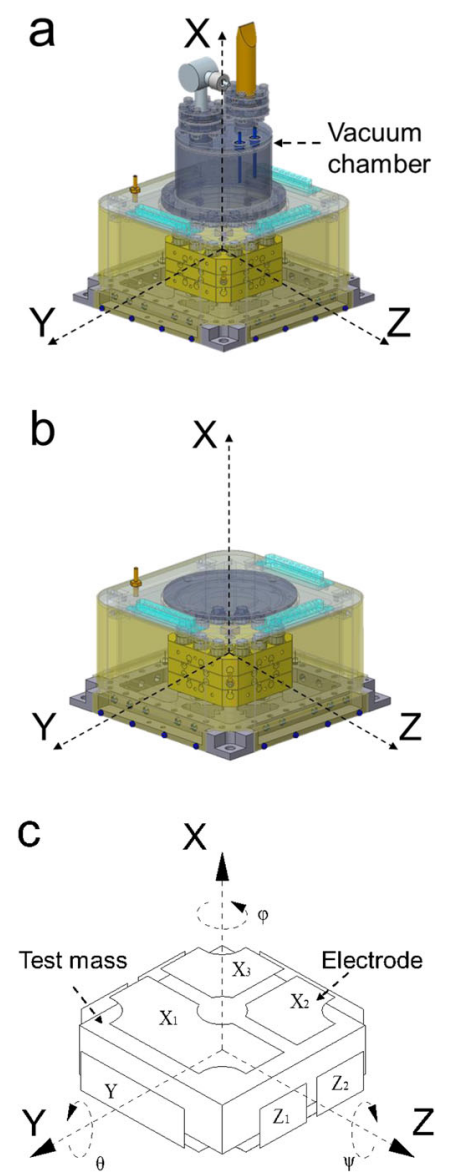

Fig. 1 The SIS and the inertial sensor. a Schematic diagram of the SIS. b Schematic diagram of the actual inertial sensor. c Axial diagram of the electrode housing.

\begin{tabular}{|llll|}
\hline \multicolumn{4}{|l}{ Table 1. Voltage settings in the different control modes. } \\
\hline Control mode & $\begin{array}{l}\text { Position detection } \\
\text { voltage }(\mathrm{V})\end{array}$ & $\begin{array}{l}\text { Bias } \\
\text { voltage }(\mathrm{V})\end{array}$ & $\begin{array}{l}\text { Maximum feedback } \\
\text { voltage }(\mathrm{V})\end{array}$ \\
\hline $\begin{array}{l}\text { Capture mode } \\
\begin{array}{l}\text { Large- } \\
\text { range mode }\end{array}\end{array}$ & 4 & 55 & 35 \\
$\begin{array}{l}\text { Small- } \\
\text { range mode }\end{array}$ & 4 & 55 & 35 \\
\hline
\end{tabular}

The displacement signal of the test mass was obtained by measuring the capacitance difference between test mass and electrodes. At the same time, a PID controller was used to ensure that the test mass was positioned at the center of the electrode housing, and the acceleration of the sensor-head was recorded through the feedback voltages. The average value of the displacement or acceleration along each axis was used to describe the translation of the sensor-head, and the corresponding difference value was used to represent the rotation of the sensor-head.

\section{RESULTS}

\section{Brief summary}

In total, 20 drop tests were conducted and a complete set of test methods was established, including a simulation model and a way to control the simulation precision. For the first time, a switch of

\section{a}

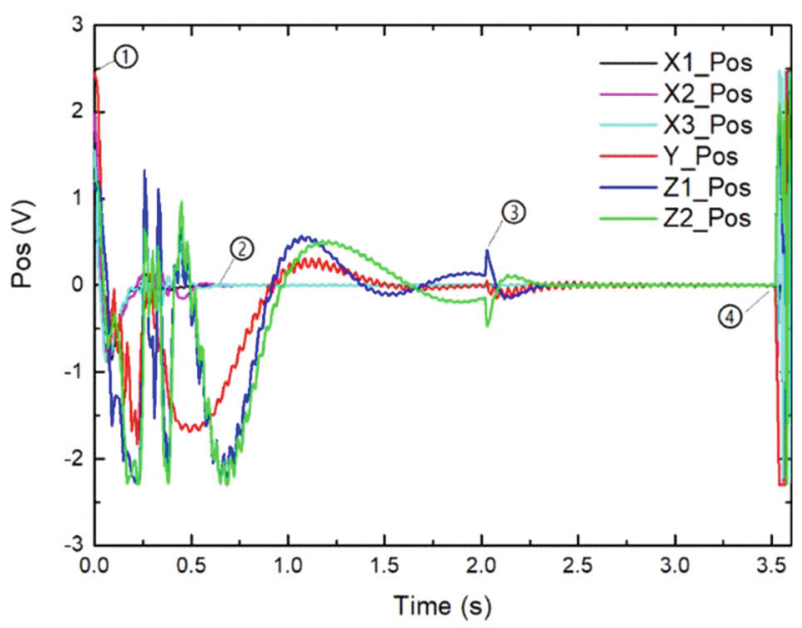

b

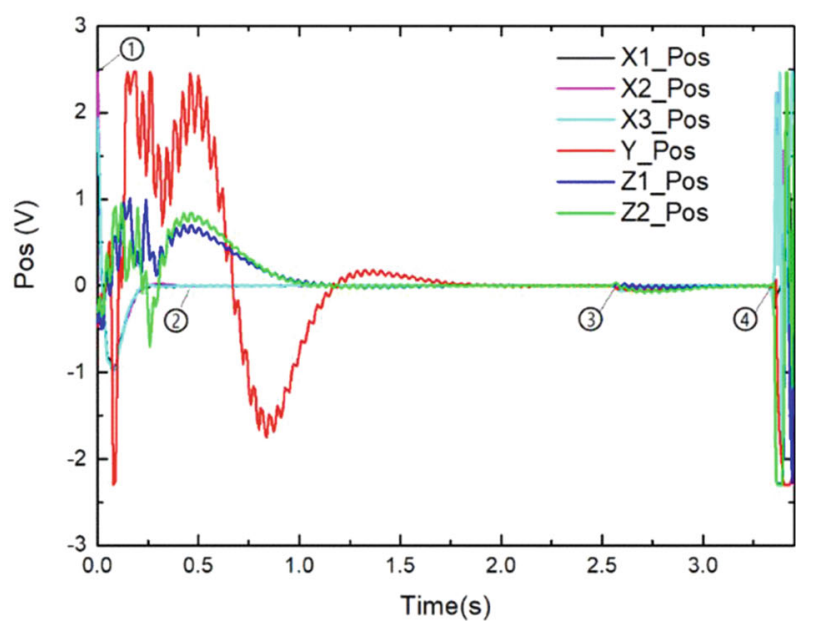

Fig. 2 Displacement voltages of all axes. In both large- and smallrange modes $(\mathbf{a}, \mathbf{b})$, the outer capsule began to fall at $(1)$, the test mass along the $\mathrm{X}$-axis reached the center of the electrode housing at (2), and the mode switch took place at (3). The free-fall ended at (4) due to collision between the inner and outer capsules.

different circuit gains was achieved in a drop tower test. As long as the control parameters were set to reasonable values, the effect of circuit gain switching on system stability was negligible. These results provide an important experimental reference for the inorbit operation of the inertial sensor on the Taiji-1 satellite. Furthermore, the NMLC drop tower's residual accelerations in three dimensions were measured. In the last $0.5 \mathrm{~s}$ of the free-fall, the lowest residual acceleration reached about $58 \mu g_{0}$ along the $X-$ axis (the falling direction) and $13 \mu \mathrm{g}_{0}$ along the $\mathrm{Y}$ - and Z-axes. The experimental results in this paper also have important reference value for researchers who plan to conduct experiments using the NMLC drop tower.

\section{Control modes}

The different control modes were named as capture mode, largerange mode, and small-range mode. The voltage parameter settings for the different control modes are given in Table 1.

Due to the fact that the duration of the free-fall was only about $3.5 \mathrm{~s}$, the time to switch from the capture mode to the large-range mode, and then to the small-range mode at one single drop was 
a

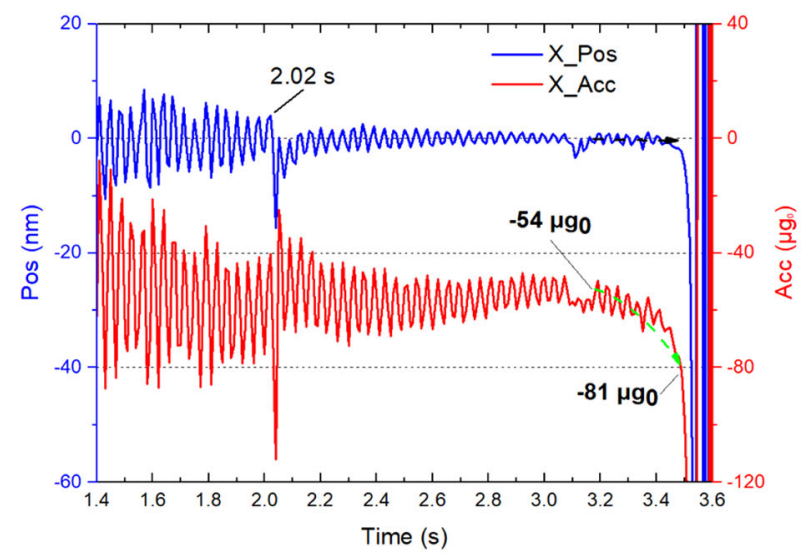

b

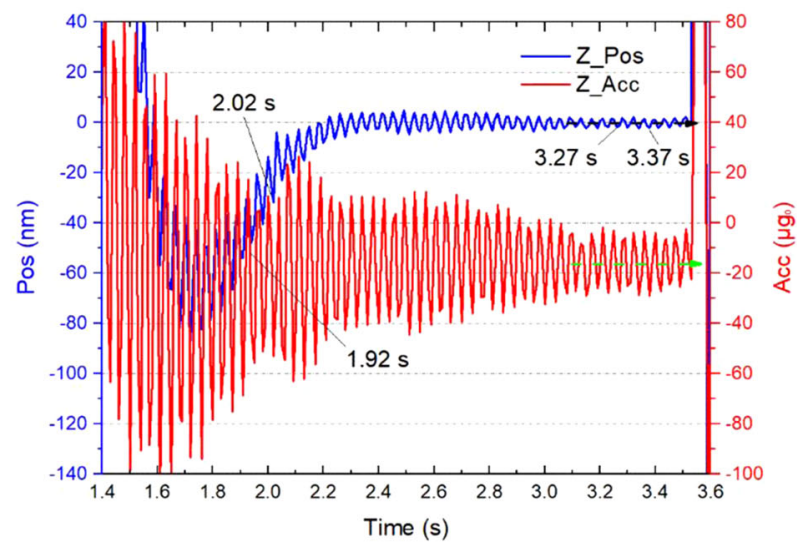

Fig. 3 Magnified view of the measured curves in the large-range mode switching experiment. The displacement (blue) and acceleration (red) along the X- and Z-axes are shown in (a) and (b), respectively. The green dashed arrows indicate the residual acceleration while the black dashed arrows indicate the displacement at the end of the free-fall.

not sufficient. To this end, two sets of experiments were designed; a large-range mode switching experiment and a small-range mode switching experiment. Each experiment involved a change from capture mode to large- or small-range mode, respectively.

\section{Modes switching}

The displacement voltage curves for each direction during the free-fall in the large-range mode switching experiment are shown in Fig. 2a. Before the free-fall, the SIS rested on mechanical stops, and the displacement voltages along each axis remained constant. At $0 \mathrm{~s}$, the outer capsule began to fall, and after $0.64 \mathrm{~s}$ (from (1) to (2), the test mass of the SIS along the X-axis was controlled to the center of the electrode housing. The displacement curves in the horizontal axes ( $\mathrm{Y}$ - and $\mathrm{Z}$-axes) converged more slowly, requiring about $1.5 \mathrm{~s}$ to converge to zero. The mode switch took place at (3) and caused a voltage overshoot of up to $0.3 \mathrm{~V}$ along the $Z 1$ and $Z 2$ axes. Then, the SIS switched to the large-range mode and the test mass reached the center of the electrode housing within $0.5 \mathrm{~s}$. The microgravity environment of the inner capsule disappeared at (4) $(3.52 \mathrm{~s})$ and the displacement voltages of each axis changed dramatically due to the collision between the inner and outer capsules.

The displacement voltage curves for each direction during the free-fall in the small-range mode switching experiment are shown in Fig. $2 \mathrm{~b}$. The displacement voltages along the $\mathrm{X}$-axis converged within $0.44 \mathrm{~s}$ (from (1) to (2), while it took a longer time for the $Y$ and Z-axes to converge to zero. At point (3, the SIS switched to small-range mode, causing a vibration of only about $0.1 \mathrm{~V}$. Then, the test mass reached the center of the electrode housing within $0.2 \mathrm{~s}$ along the $\mathrm{X}$-axis and within about $0.75 \mathrm{~s}$ along the horizontal axes. The free-fall lasted about $3.32 \mathrm{~s}$ (from (1) to (4)).

\section{DISCUSSION}

The translational motion of the SIS was determined by calculating the average displacement and acceleration along each axis. The displacement and acceleration along the $\mathrm{X}$-axis are presented in Fig. 3a. The mode switching took place at $2.02 \mathrm{~s}$ and the displacement-detection gain was reduced by four times compared to that in capture mode. The PID parameters changed accordingly. This induced a displacement vibration of about $20 \mathrm{~nm}$ only and an acceleration overshoot of about $87 \mu g_{0}$, which was attributed to the change of the electrostatic forces applied to the test mass. At the end of the free-fall, a significant increase in acceleration occurred (green dashed arrow), while the test mass was maintained at the center of the electrode housing (black dashed arrow) until the last $0.1 \mathrm{~s}$.

A magnified view of the displacement and acceleration along the Z-axis before and after mode switching is presented in Fig. $3 \mathrm{~b}$. As indicated by the dashed arrows, both the displacement and acceleration along the Z-axis remained stable until the last $0.1 \mathrm{~s}$. This is in sharp contrast to the X-axis data presented in Fig. 3a. This difference may have been induced by the air resistance during the free-fall or by the interaction between the inner and outer capsules during the end of the free-fall.

The air-drag force can be expressed as Eq. (1): ${ }^{20}$

$F=\frac{1}{2} C_{\mathrm{D}} \rho S v^{2}$

where $F$ is the air-drag force, $C_{D}$ is the drag coefficient, $\rho$ is the air density, $S$ is the cross-sectional area of the capsule, and $v$ is the velocity relative to the atmosphere.

The residual acceleration of the inner capsule can be expressed as Eq. (2): ${ }^{20}$

$a_{\mathrm{r}}=\mathrm{g}_{0} \tanh ^{2} \sqrt{\frac{C_{\mathrm{D}} \rho S \mathrm{~g}_{0} t}{2 m}}$

Based on the parameters of the inner capsule (i.e., $m=217 \mathrm{~kg}$, $C_{D}=0.2, \rho=1.15 \mathrm{e}-3 \mathrm{~kg} / \mathrm{m}^{3}$, and $S=0.385 \mathrm{~m}^{2}$ ), the acceleration induced by air drag and that measured along the $X$ - and Z-axes in the large-range mode switching experiment were compared, and the comparison results are presented in Fig. 4a. Compared with the rapid acceleration change along the $X$-axis in the last $0.3 \mathrm{~s}$, the amplitude of the air-drag acceleration was much smaller and increased more slowly. Therefore, it can be confirmed that the rapid acceleration change along the $X$-axis was not induced by the air resistance.

Seventy-two magnetic beads were placed at the bottom of the outer capsule (Fig. 4b), which were used to attract and fix the inner capsule at the end of the free-fall, and prevent the collision between the outer and inner capsules after contacting the deceleration unit.

At the last $0.3 \mathrm{~s}$ of the free-fall, the inner capsule reached near the bottom of the outer capsule before the latter touched the deceleration unit. Consequently, it can be inferred that the 72 magnetic beads attracted the inner capsule and induced a large acceleration change along the $\mathrm{X}$-axis.

The data within $0.1 \mathrm{~s}$ in the most stable period in both the capture mode (1.92 2.02 s) and the large-range mode (3.27 3.37 s) exhibited the minimum displacement and lowest acceleration. The average value and maximum fluctuations were calculated, and are shown in Table 2. 

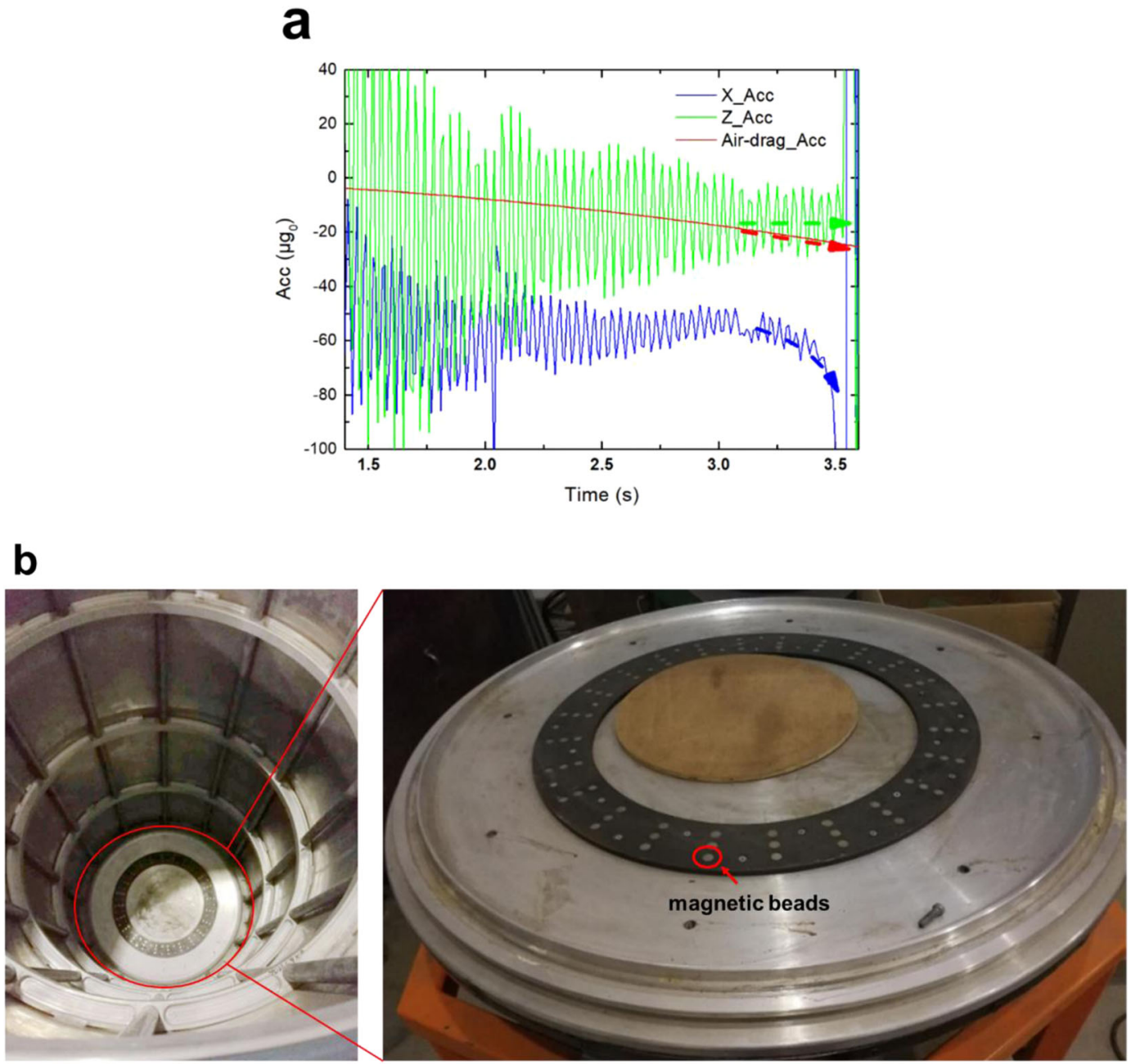

Fig. 4 Analysis of the end of free-fall. a Comparison between the acceleration induced by air drag (red) and that measured along the X-and Z-axes (blue and green) in the large-range mode switching experiment. $\mathbf{b}$ Top view of the outer capsule (left) and closer view of the bottom of the outer capsule, where the distribution of the magnetic beads can be observed (right).

\begin{tabular}{|c|c|c|c|c|c|}
\hline \multirow[t]{2}{*}{ Control mode } & \multirow[t]{2}{*}{ Test item } & \multicolumn{2}{|c|}{ Displacement (nm) } & \multicolumn{2}{|c|}{$\begin{array}{l}\text { Acceleration } \\
\left(\mu \mathrm{g}_{0}\right)\end{array}$} \\
\hline & & $X$ & Z & $\mathrm{X}$ & Z \\
\hline \multirow[t]{2}{*}{ Capture mode } & Average value & 0.3 & -30.4 & -59.3 & -20.5 \\
\hline & $\begin{array}{l}\text { Maximum } \\
\text { fluctuations }\end{array}$ & 8.6 & 34.3 & 38.2 & 73.3 \\
\hline \multirow{2}{*}{$\begin{array}{l}\text { Large- } \\
\text { range mode }\end{array}$} & Average value & -0.3 & -0.1 & -59.4 & -15.5 \\
\hline & $\begin{array}{l}\text { Maximum } \\
\text { fluctuations }\end{array}$ & 3.1 & 4.5 & 12.8 & 25.6 \\
\hline \multicolumn{2}{|c|}{$\begin{array}{l}\text { Largest vibration during mode } \\
\text { switching }\end{array}$} & 20 & 28 & 87 & 63 \\
\hline
\end{tabular}

After mode switching, the average acceleration along the Z-axis was reduced from -20.5 to $-15.5 \mu \mathrm{g}_{0}$ and the acceleration fluctuation was reduced by about three times from 73.3 to $25.6 \mu \mathrm{g}_{0}$, indicating that the test mass was further controlled and the average displacement reached $-0.1 \mathrm{~nm}$ only (Table 2 ).

In the small-range mode switching experiment, the mode switch of the $\mathrm{X}$-axis took place at $2.54 \mathrm{~s}$, producing an obvious vibration (Fig. 5a). The displacement-detection gain was reduced by four times and the feedback-loop gain was reduced by half after the switch to small-range mode.

The magnified views of $Y$ - and Z-axes are illustrated in Fig. 5b, c, respectively. The displacement and acceleration data along both axes remained stable before the end of the free-fall, as indicated by the green boxes.

The data along the three axes within $0.1 \mathrm{~s}$ in the most stable period in the capture mode (2.44 2.54 s) and small-range mode (2.92 3.02 s) are presented in Table 3.

Based on the average acceleration of the X-axis in both largeand small-range modes, we conclude that during the free-fall test, the minimum residual acceleration along the $\mathrm{X}$-axis was about $-58 \mu \mathrm{g}_{0}$ and about $-13 \mu \mathrm{g}_{0}$ along the horizontal axes. Furthermore, the largest acceleration vibration was about $87 \mu g_{0}$ (Table 2) and the displacement vibration was only about $35 \mathrm{~nm}$ (Table 3) during mode switching, indicating that when the control parameters are set properly, the effect of circuit gain switching on system stability is negligible.

The inner capsule was equipped with a uniaxial quartzaccelerometer (QA), which can measure the acceleration along the falling direction. The resolution of the QA was $4.675 \mathrm{mg}_{0}$, and the measurement range was $\pm 7.7 \mathrm{~g}_{0}$. The acceleration measured by the QA can record the state of the inner capsule during the free-fall. 
a

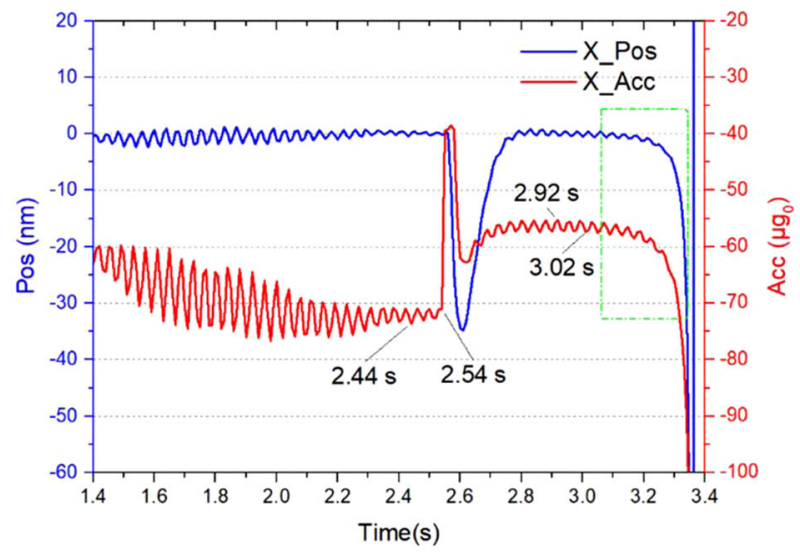

b

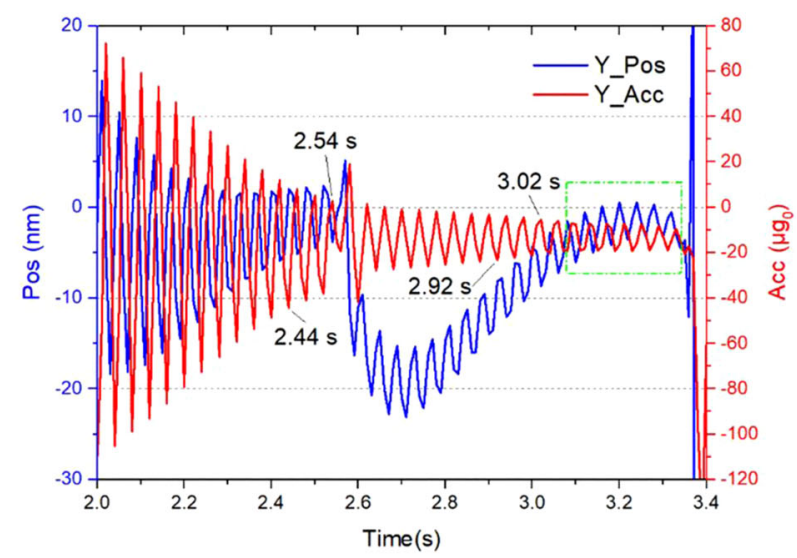

C

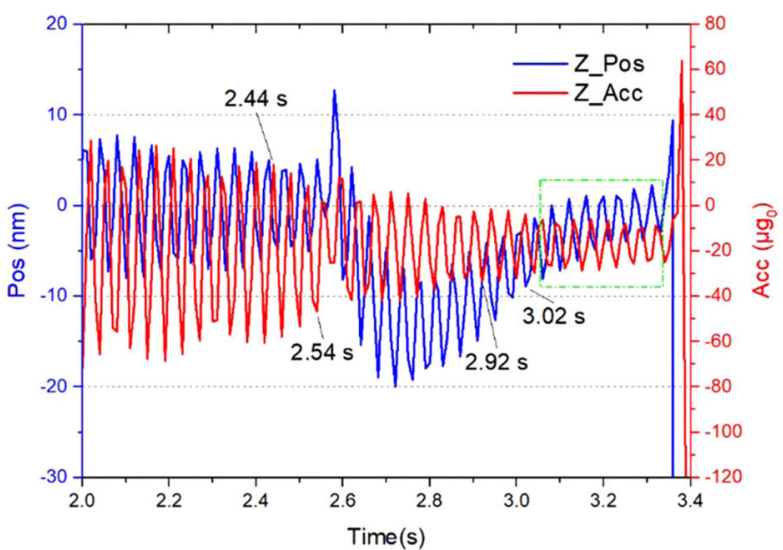

Fig. 5 Magnified view of the measured curves in the small-range mode switching experiment. The displacement (blue) and acceleration (red) along $X_{-}, Y_{-}$, and Z-axes are shown in (a), (b), and (c), respectively. The green dashed box indicates the end of the free-fall.

The displacement data measured by the SIS along the X2 axis and the acceleration data measured by the QA in the large-range mode switching test were chosen for comparison (Fig. 6a, b).

Before $0 \mathrm{~s}$, the microgravity level measured by the QA along the falling direction was about $1 \mathrm{~g}_{0}$, while the displacement voltage of the SIS was at a saturation state, indicating that the inner capsule
Table 3. Comparison of displacement and acceleration data in the small-range mode switching experiment.

\begin{tabular}{|c|c|c|c|c|c|c|c|}
\hline \multirow[t]{2}{*}{ Control mode } & \multirow[t]{2}{*}{ Test item } & \multicolumn{3}{|c|}{ Displacement $(\mathrm{nm})$} & \multicolumn{3}{|c|}{ Acceleration $\left(\mu g_{0}\right)$} \\
\hline & & $\mathrm{x}$ & $\mathrm{Y}$ & Z & $\mathrm{x}$ & $\mathrm{Y}$ & Z \\
\hline \multirow[t]{2}{*}{ Capture mode } & Average value & 0.1 & -1.0 & -0.3 & -72.0 & -16.4 & -21.2 \\
\hline & $\begin{array}{l}\text { Maximum } \\
\text { fluctuations }\end{array}$ & 0.7 & 7.5 & 10.1 & 2.7 & 52.5 & 76.3 \\
\hline \multirow{2}{*}{$\begin{array}{l}\text { Small- } \\
\text { range mode }\end{array}$} & Average value & -0.01 & -8.1 & -7.2 & -56.4 & -13.1 & -17.6 \\
\hline & $\begin{array}{l}\text { Maximum } \\
\text { fluctuations }\end{array}$ & 1.0 & 7.7 & 9.8 & 2.0 & 19.5 & 31.7 \\
\hline \multicolumn{2}{|c|}{$\begin{array}{l}\text { Largest vibration during mode } \\
\text { switching }\end{array}$} & 35 & 28 & 33 & 32 & 61 & 48 \\
\hline
\end{tabular}

a

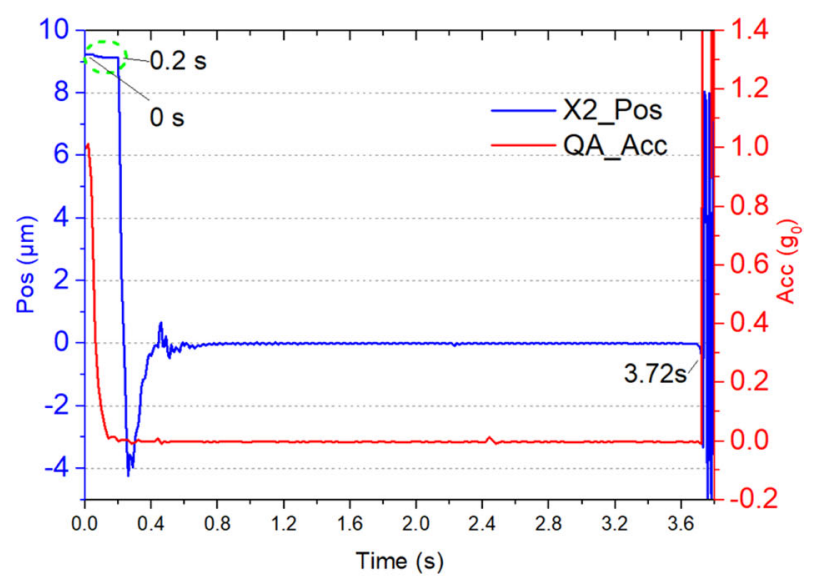

b

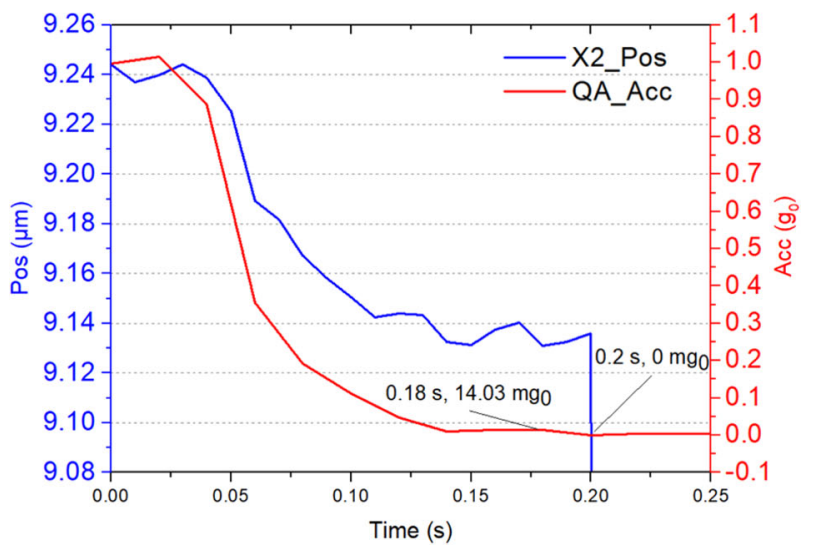

Fig. 6 Data comparison between the displacement along the X2 axis and the acceleration of the $Q A$ in the large-range mode switching experiment. a Data of the X2 axis (blue) and the QA (red) during the free-fall. $\mathbf{b}$ Magnified view at the beginning of the release of the outer capsule $(0 \sim 0.25 \mathrm{~s})$.

was stationary at the top of the tower. As soon as the outer capsule was released at $0 \mathrm{~s}$, the acceleration measured by the QA decreased rapidly towards zero (Fig. 6a). Meanwhile, the displacement of the test mass changed by only about $0.1 \mu \mathrm{m}$ in the first $0.2 \mathrm{~s}$, which was caused by the vibration generated during the release of the inner capsule. Although the residual acceleration decreased rapidly during the first $0.2 \mathrm{~s}$ of the free-fall, it still 


\begin{tabular}{|c|c|c|c|c|c|}
\hline \multirow[t]{3}{*}{ Axis } & \multicolumn{3}{|l|}{ SIS } & \multicolumn{2}{|c|}{ Inertial sensor } \\
\hline & \multirow{2}{*}{$\begin{array}{l}\text { Maximum } \\
\text { operational } \\
\text { acceleration } \\
\left(\mathrm{mg}_{\mathrm{o}}\right)\end{array}$} & \multicolumn{2}{|l|}{ Range } & \multirow{2}{*}{$\begin{array}{l}\text { Maximum } \\
\text { operational } \\
\text { acceleration } \\
\left(\mathrm{mg}_{0}\right)\end{array}$} & \multirow[t]{2}{*}{ Range } \\
\hline & & $\begin{array}{l}\text { Large- } \\
\text { range mode }\end{array}$ & $\begin{array}{l}\text { Small- } \\
\text { range mode }\end{array}$ & & \\
\hline $\mathrm{X}$ & 9.60 & 15.0 & 4.30 & 1.60 & 0.04 \\
\hline Y & 0.93 & 1.6 & 0.46 & 0.18 & 0.005 \\
\hline Z & 0.93 & 1.6 & 0.46 & 0.18 & 0.006 \\
\hline
\end{tabular}

a

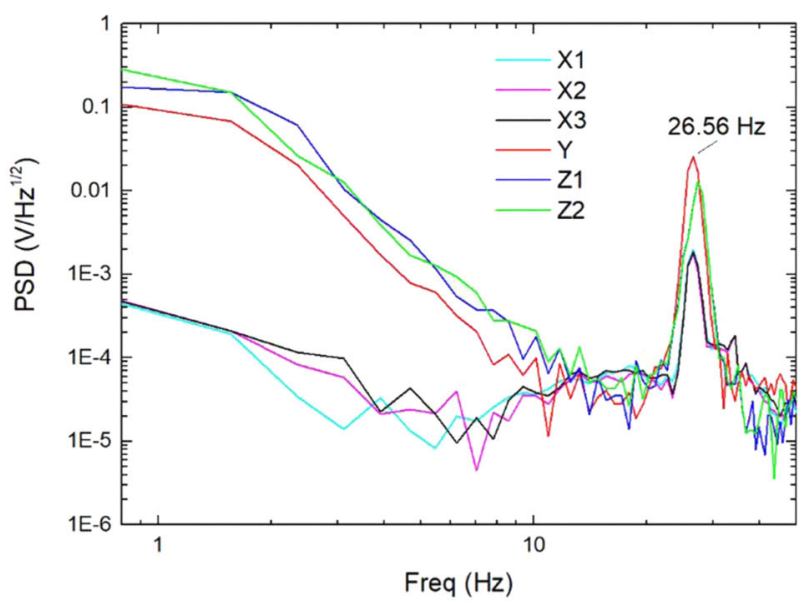

b

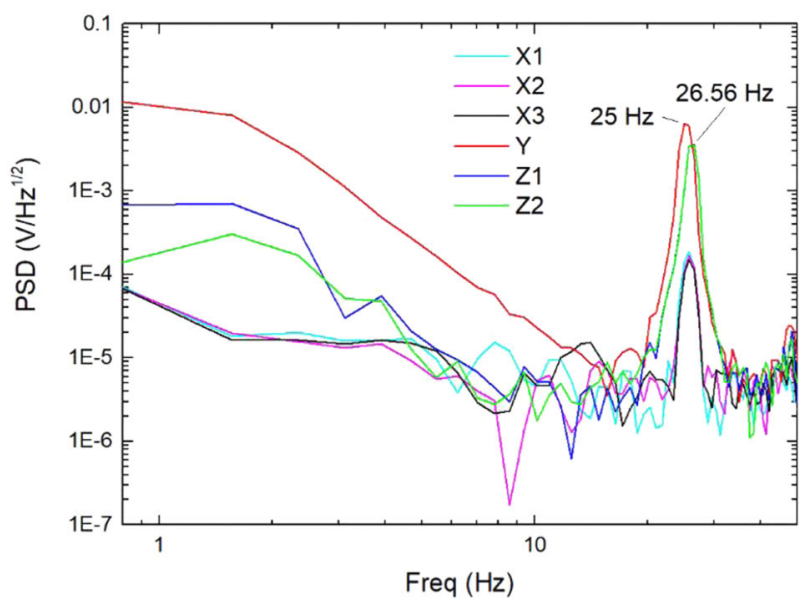

Fig. 7 Spectrum analyses. Resonance peak of the SIS displacement voltages in the large- and small-range mode switching experiments (a, b).

exceeded the highest capture-acceleration in the X-axis of the SIS (9.6 mgo; Table 4) and the test mass could not reach the center of the electrode housing. At $0.2 \mathrm{~s}$, the acceleration measured by the $Q A$ reached zero (Fig. $6 \mathrm{~b}$ ), indicating that the residual acceleration was less than $4.675 \mathrm{mg}_{0}$ (resolution of the QA), and lower than the capture-acceleration of the SIS along the X-axis; thus, the test mass moved quickly towards the center of the electrode housing.

Owing to the disturbance during the release of the capsule, the residual acceleration along the $\mathrm{X}$-axis would be higher than
$10 \mathrm{mg}_{0}$ in a short time (e.g., $0.2 \mathrm{~s}$ ), and the test mass could not be captured in this period. A conclusion that can be drawn is that in order to achieve the longest free-fall time in a drop tower test, a stable release process with minimum residual acceleration should be ensured. For instance, under the premise of synchronous release of the outer and inner capsules, experiments should not be performed in the case of strong external winds (greater than $10 \mathrm{~m} / \mathrm{s}$ ) in order to avoid additional disturbance from the drop tower. In addition, strong rigid connections should be used for the inner capsule to reduce the vibration after the release. At the same time, all equipment and cables should be firmly fastened to the capsule.

Spectrum analyses for both the large- and small-range mode switching experiments were performed. The SIS displacement voltages in the capture mode were used and the results are shown in Fig. $7 a, b$.

The acceleration of the inner capsule increased abruptly from 0 to $1 \mathrm{~g}_{0}$ when the electromagnetic lock powered off, which caused the inner capsule to vibrate near its natural resonant frequency. As can be seen in Fig. 7a, b, there were apparent resonance peaks around $26.56 \mathrm{~Hz}$ in each axial direction, which reflect the natural resonant frequency of the inner capsule.

\section{METHODS}

\section{NMLC drop tower and experimental equipment}

The drop tower of the NMLC in Beijing (Fig. 8a) has a total height of $124 \mathrm{~m}$, including $116 \mathrm{~m}$ above the ground and $8 \mathrm{~m}$ below the ground. The total free-fall distance is about $60 \mathrm{~m}$, providing a free-fall time of about $3.5 \mathrm{~s}$. Figure $8 \mathrm{~b}$ illustrates a sketch of the drop tower experimental configuration. The X-axis of the SIS was parallel to the longitudinal axis of the inner capsule, while the $\mathrm{Y}$ - and Z-axes of the SIS were perpendicular to the $\mathrm{X}$ axis.

There are two installation modes in a drop tower test, namely, the Single Capsule Mode and the Double Capsule Mode. The microgravity level during the fall is about $10^{-3} \mathrm{~g}_{0}$ in the Single Capsule Mode and can reach about $10^{-5} \mathrm{~g}_{0}$ in the Double Capsule Mode ${ }^{19,21}$. For the experiments in this paper, the Double Capsule Mode was adopted.

\section{Drop tower test procedure}

The basic steps of the experimental process are shown in Fig. 9a. After the first self-check, all devices were installed symmetrically near the central axis of the mounting plate, which is made of wood and aluminum. All cables were tightened to eliminate any micro-vibration which would cause non-negligible interference to the measured acceleration (Fig. 9b). After completing the second self-check, the inner capsule was sealed and lifted up for barycentric correction (Fig. 9c). Additionally, by correcting the barycenter, the inner capsule was prevented from rotating during the freefall.

Subsequently, when the barycentric correction was completed, the inner capsule was lifted into the outer capsule (Fig. 9d). Then, the outer capsule was pumped below $30 \mathrm{~Pa}$ and sealed (Fig. 9e). Before falling from the top of the tower, the vacuum pressure generally increased to around $80 \mathrm{~Pa}$ due to the slow air leakage effect.

Data were recorded from the beginning to the end of the experiment until the power of the inner capsule was cut off. In order to ensure that the power during the test was substantial, both the MCU and the data acquisition card were powered independently by lead batteries.

\section{Control strategy and parameter settings}

In order to adapt to the microgravity level in the NMLC drop tower $\left(10^{-2}\right.$ $\mathrm{mg}_{\mathrm{o}}$ ), the maximum feedback voltages of the SIS were amplified and the measurement range along the horizontal axes was expanded from $\pm 5 \mu \mathrm{g}_{0}$ to $\pm 1.6 \mathrm{mg}_{0}$ (large-range mode) and $\pm 0.46 \mathrm{mg}_{0}$ (small-range mode). At the same time, the ability to capture the test mass in a microgravity environment was enhanced, ensuring an adequate capturing and measurement ability for the drop tower experiment (Table 4). The maximum operational acceleration reflects the maximum residual acceleration at which the SIS can operate. 

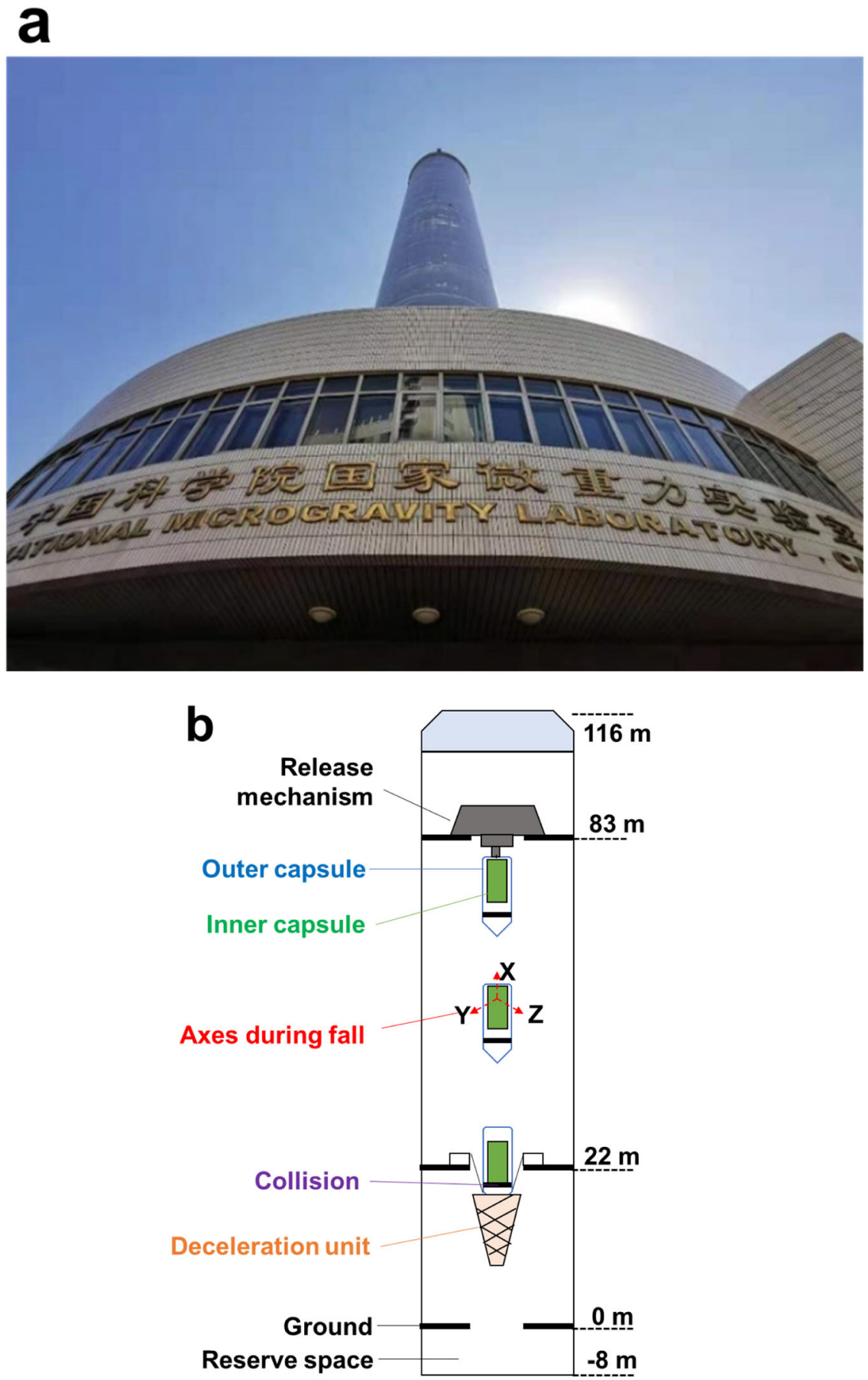

Fig. 8 The NMLC drop tower. a An upward view of the building. b Sketch of the drop tower's experimental configuration.

At the beginning of the free-fall, the test mass in the SIS rested on the bottom of the electrode housing. The primary task was to control the test mass to the center of the electrode housing. Therefore, prior to measurement, the MCU was in capture mode with the lowest position detection voltage $(1 \mathrm{~V})$, the highest bias voltage $(55 \mathrm{~V})$, and a maximum feedback voltage of up to $35 \mathrm{~V}$. In this parameter configuration, the maximum operational acceleration was relatively large (Table 4).

Once the displacement voltages of all axes were within $\pm 0.5 \mathrm{~V}$ and the duration exceeded $0.5 \mathrm{~s}$, the large-range mode switching would begin and $V_{\mathrm{d}}$ would switch from 1 to $4 \mathrm{~V}$ to increase the stiffness and optimize the stability of the system.

In the small-range mode switching experiment, the mode switch would begin when the displacement voltages of all axes were within $\pm 0.1 \mathrm{~V}$ for more than $0.5 \mathrm{~s}$. After the mode switch, the bias voltage was reduced from
55 to $30 \mathrm{~V}$, the gain of the feedback loop was reduced by half, and the position detection voltage $\left(V_{\mathrm{d}}\right)$ was switched to $4 \mathrm{~V}$.

By reducing the bias voltage and the gain of the feedback loop, the circuit noise was suppressed, improving the resolution of the measurement. This small-range mode allows for more accurate measurements when the residual acceleration of the satellite is further reduced.

\section{Mode switch simulation prior to free-fall}

A single drop tower experiment may last $4 \sim 5 \mathrm{~h}$ and may fail for unpredictable reasons. To ensure the success of the experiment, the Simulink software (https://ww2.mathworks.cn) was used to simulate the control stability of the system during the fall and determine the optimal control parameters before and after the mode switch. 


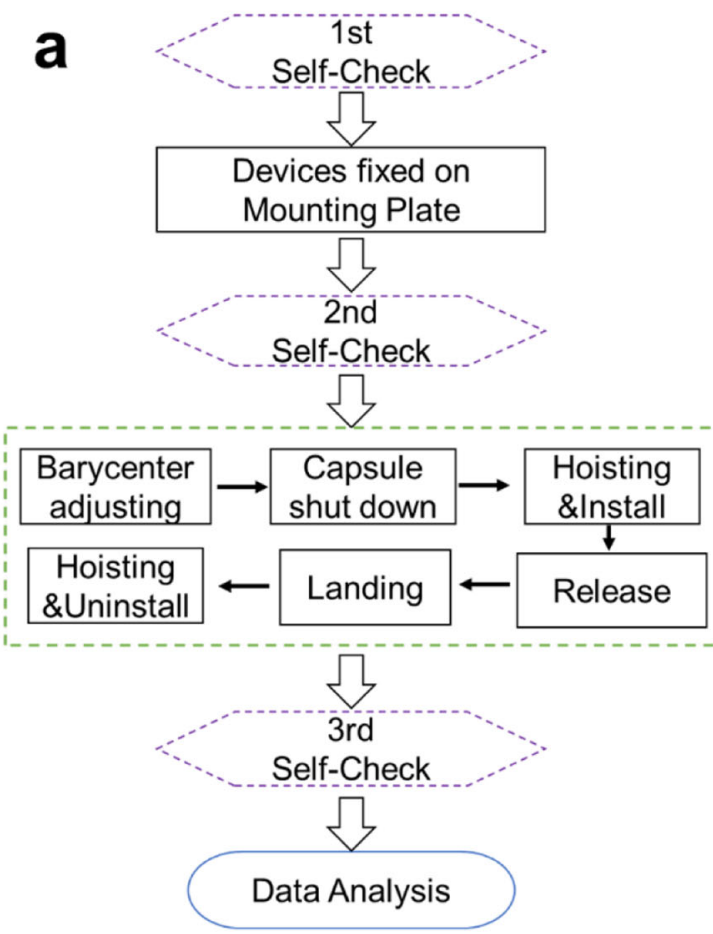

b

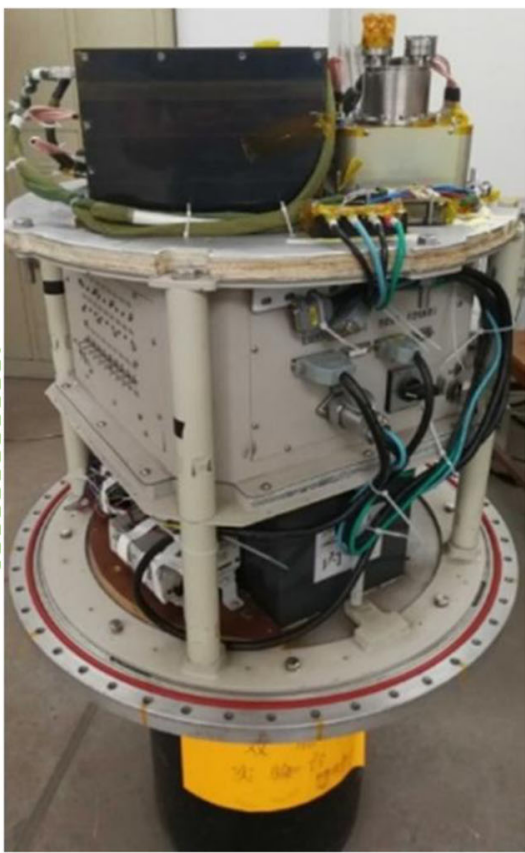

c

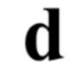

$\mathbf{e}$

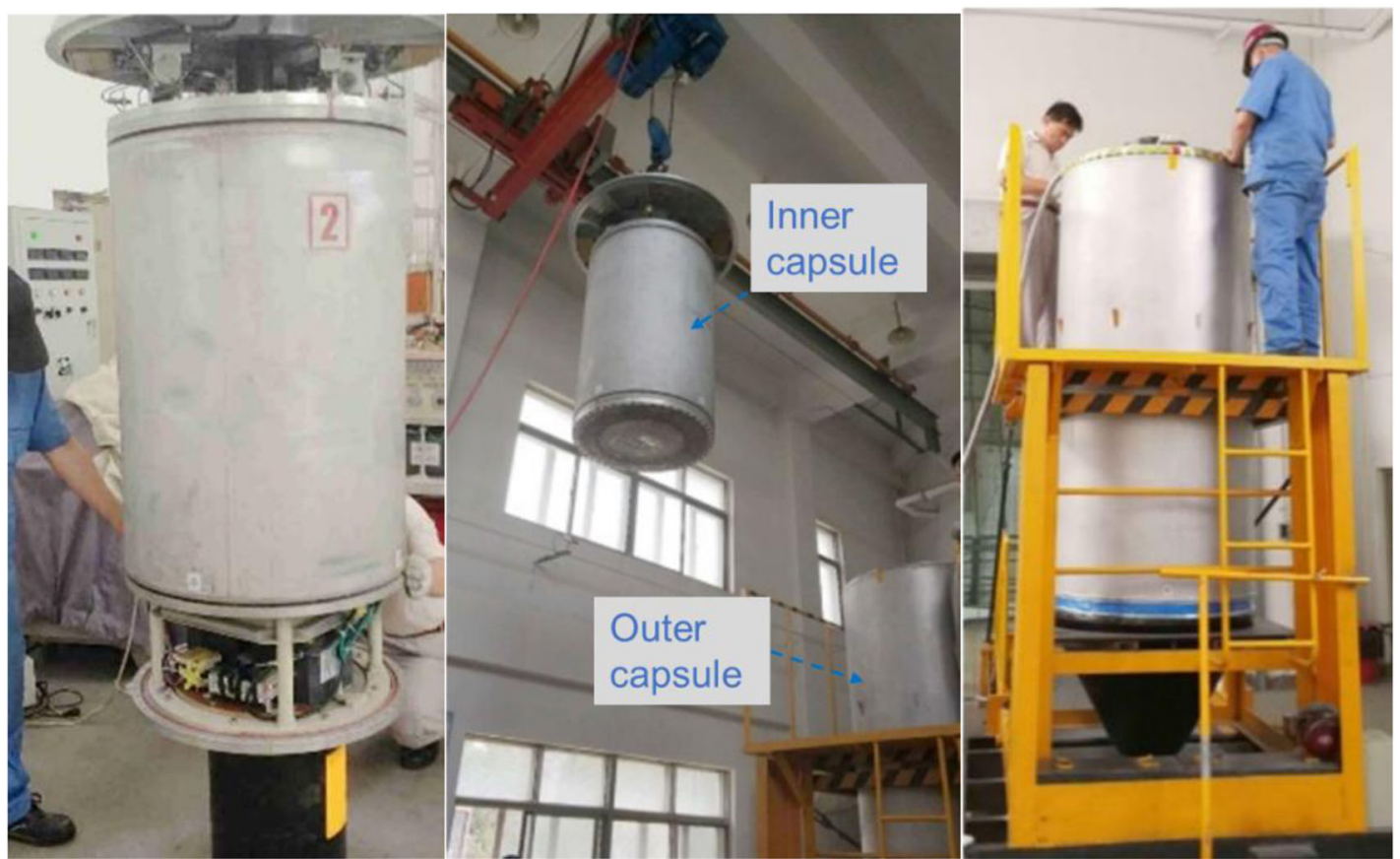

Fig. 9 Drop tower test procedure. a Flowchart of the drop tower test. b Devices installed on the mounting plate. c Sealed inner capsule for barycentric correction. $\mathbf{d}$ Lifted inner capsule into the outer capsule. e Outer capsule pumped below 30 Pa and sealed.

The uniaxial simulation model for the drop tower test is illustrated in Fig. $10 \mathrm{a}$, where $a_{\text {ext }}$ is the external residual acceleration, $x_{\text {mass }}$ is the displacement of the test mass, $U_{0}$ is the output voltage of the charge amplifier, $V_{\mathrm{f}}$ is the feedback voltage, and $a_{\text {mass }}$ is the acceleration produced by the electrostatic force.

In the simulation model, the noise, gain, and delay in the real circuit were taken into consideration. According to the microgravity level of the drop tower ${ }^{21}, a_{\text {ext }}$ was set to a constant value of $-2 \times 10^{-3} \mathrm{~m} / \mathrm{s}^{2}$ along the three axes of the model.

The PID controller plays a crucial role in a real circuit, since, when inorbit, the test mass may get out of control if the key parameters of the PID controller are not set properly. The available time is limited since there are only about $3.5 \mathrm{~s}$ of free-fall during a drop test; thus, the effectiveness and correctness of the control loop are of particular importance. 
a

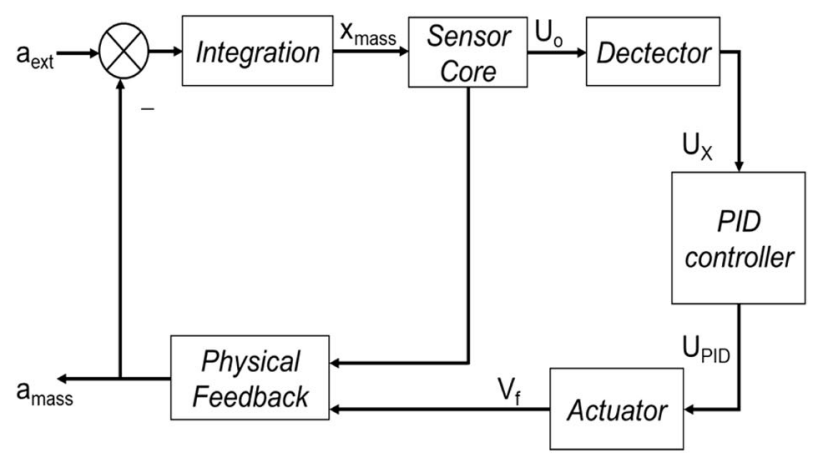

b

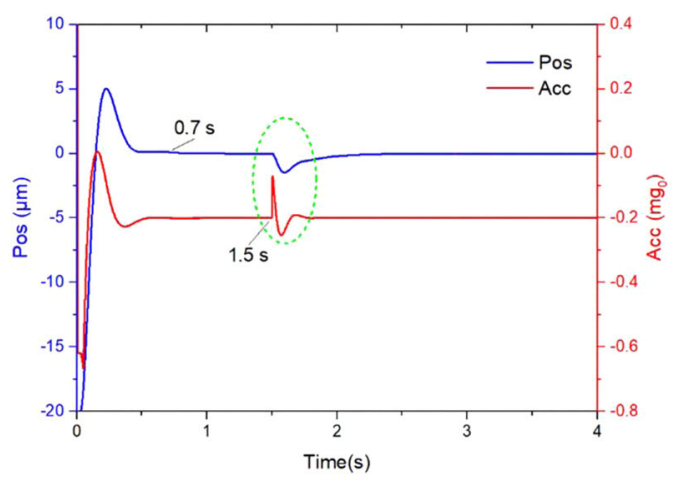

Fig. 10 Simulation for the drop tower test. a Uniaxial simulation model of the SIS. $\mathbf{b}$ Displacement and acceleration along the $\mathrm{Y}$-axis in small-range mode switching experiment.

The PID controller contains proportional, differential, and integral terms. Its transfer function ${ }^{22,23}$ is given by Eq. (3).

$G(s)=K_{\mathrm{p}}+\frac{K_{\mathrm{l}}}{s}+K_{\mathrm{D}} s$

where $K_{\mathrm{p}}$ is the proportionality coefficient, $K_{\mathrm{l}}$ is the integral term coefficient, $K_{\mathrm{D}}$ is the differential term coefficient, and $s$ is the Laplace operator.

In the simulation, $K_{\mathrm{p}}, K_{\mathrm{l}}$, and $K_{\mathrm{D}}$ need to be fine-tuned so that a sufficient gain and phase margin can be obtained for a stable system.

In the uniaxial simulation model, an electrostatic force equation was used, as shown in Eq. (4) ${ }^{24}$.

$F_{\mathrm{el}}=2 \varepsilon_{0} S \frac{V_{\mathrm{p}} V_{\mathrm{f}}\left(d^{2}+x^{2}\right)-\left(V_{\mathrm{f}}^{2}+V_{\mathrm{p}}^{2}+V_{\mathrm{d}}^{2}\right) \cdot d \cdot x}{\left(d^{2}-x^{2}\right)^{2}}$

where $S$ is the area of the electrode, $\varepsilon_{0}$ is the permittivity of vacuum, $V_{p}$ is the bias voltage, $V_{\mathrm{f}}$ is the feedback voltage, $V_{\mathrm{d}}$ is the detection voltage, $d$ is the average distance between electrode and test mass when the test mass is at the center of the electrode housing, and $x$ is the displacement of test mass relative to the center of the electrode housing.

According to Eq. (4), the electrostatic force applied on the test mass can be calculated from the real-time feedback voltage $\left(V_{f}\right)$ and displacement $(x)$.

The simulation results of the displacement and acceleration along the $Y$ axis in the small-range mode switching test were taken as an example, as shown in Fig. 10b. It can be seen that at about $0.7 \mathrm{~s}$ after release, the test mass can be controlled at the center of the electrode housing. The mode switch took place at $1.5 \mathrm{~s}$, causing a vibration of up to $0.55 \mu \mathrm{m}$, and an acceleration overshoot of about $0.21 \mathrm{mg}_{0}$, as indicated by the circled area. After mode switching, the displacement curve converged to zero within $0.7 \mathrm{~s}$.

The other axes were simulated and optimized in the same way to ensure that the test mass could be captured within $1 \mathrm{~s}$ and the displacement vibration would remain within $\pm 1 \mu \mathrm{m}$ after mode switching. The experimental results show that a proper simulation before a drop test is a prerequisite for the success of the test.

\section{Reporting summary}

Further information on research design is available in the Nature Research Reporting Summary linked to this article.

\section{DATA AVAILABILITY}

The datasets generated and analyzed during the current study are available from the corresponding author on reasonable request.

Received: 10 April 2020; Accepted: 11 June 2021;

Published online: 07 July 2021

\section{REFERENCES}

1. Abbott, B. P. et al. Observation of gravitational waves from a binary black hole merger. Phys. Rev. Lett. 116, 061102 (2016).

2. Abbott, B. P., Jawahar, S., Lockerbie, N. A. \& Tokmakov, K. V. LIGO Scientific Collaboration and Virgo Collaboration (2016) GW150914: first results from the search for binary black hole coalescence with Advanced LIGO. Phys. Rev. D. 93, 122003 (2016).

3. Schutz, B. F. Gravitational-wave sources. Class. Quantum Grav. 13, A219-A238 (1996).

4. Danzmann, K. et al. LISA: Unveiling a Hidden Universe. Report No. ESA/SRE 3 (European Space Agency, 2011).

5. Danzmann, K. et al. Laser Interferometer Space Antenna. LISA L3 Mission Proposal: In response to call by the European Space Agency ESA for L3 mission concepts. https://www.elisascience.org/files/publications/LISA_L3_20170120.pdf (2017).

6. Armano, M. et al. Sub-femto-g free fall for space-based gravitational wave observatories: LISA Pathfinder results. Phys. Rev. Lett. 116, 231101 (2016).

7. Hu, W. R. \& Wu, Y. L. The Taiji Program in space for gravitational wave physics and the nature of gravity. Natl Sci. Rev. 4, 685-686 (2017).

8. Luo, J. et al. TianQin: a space-borne gravitational wave detector. Class. Quantum Grav. 33, 035010 (2016).

9. Ruan, W. H., Guo, Z. K., Cai, R. G. \& Zhang, Y. Z. et al. Taiji program: Gravitationalwave sources. Int. J. Modern Phys. A 2050075 (2020).

10. Luo, Z. R., Guo, Z. K., Jin, G., Wu, Y. L. \& Hu, W. R. A brief analysis to Taiji: science and technology. Results Phys. 16, 102918 (2020).

11. Luo, Z. R., Liu, H. S. \& Jin, G. The recent development of interferometer prototype for Chinese gravitational wave detection pathfinder mission. Opt. Laser Technol. 105, 146-151 (2018)

12. He, J. W. et al. Experimental study of a mini inductively coupled radio-frequency plasma neutralizer. J. Propuls. Technol. 39, 1673-1680 (2018).

13. Li, Y. P. et al. Demonstration of an ultraprecise optical bench for the Taiji space gravitational wave detection pathfinder mission. Appl. Sci. 9, 2087 (2019).

14. Touboul, P. \& Foulon, B. Space accelerometer development and drop tower experiments. Space Forum 4, 145-165 (1998).

15. Bortoluzzi, D., Foulon, B., Garcı'a Marirrodriga, C. \& Lamarre, D. Object injection in geodesic conditions: in-flight and on-ground testing issues. Adv. Space Res. $\mathbf{4 5}$ 1358-1379 (2010).

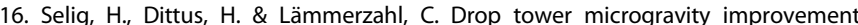
towards the nano-g level for the MICROSCOPE payload tests. Microgravity Sci. Technol. 22, 539-549 (2010).

17. Liorzou, F., Boulanger, D., Rodrigues, M., Touboul, P. \& Selig, H. Free fall tests of the accelerometers of the MICROSCOPE mission. Adv. Space Res. 54, 1119-1128 (2014).

18. Christophe, B. et al. Status of development of the future accelerometers for next generation gravity missions. In International Symposium on Advancing Geodesy in a Changing World Vol. 149 (eds Freymueller, J.T. \& Sánchez, L.) 85-89 (Springer, 2018).

19. Zhang, X. Q., Yuan, L. G., Wu, W. D., Tian, L. Q. \& Yao, K. Z. H. Some key technics of drop tower experiment device of National Microgravity Laboratory (China) (NMLC). Sci. China Ser. E-Eng. Mater. Sci. 48, 305-316 (2005).

20. Xia, Y. Microgravity drop tower test technology and its application (I). Struct. Environ. 38, 22-31 (1995).

21. Liu, T. Y., Wu, Q. P., Sun, B. Q. \& Han, F. T. Microgravity level measurement of the Beijing drop tower using a sensitive accelerometer. Sci. Rep. 6, 1-9 (2016).

22. Mei, X. R. Principles of Automatic Control. Ch. 3 (Science Press, Beijing, 2005).

23. Ang, K. H., Chong, G. \& Li, Y. PID control system analysis, design, and technology. IEEE Trans. Control Syst. Technol. 13, 559-576 (2005).

24. Xue, D. T. Servo-control analysis of electrostatically suspended accelerometer. Chin. J. Space Sci. 29, 102-106 (2009). 


\section{ACKNOWLEDGEMENTS}

The authors gratefully acknowledge support by the Pre-research Project on Civil Aerospace Technologies (A0301) of CNSA.

\section{AUTHOR CONTRIBUTIONS}

J.M. is the primary author of this paper, and performed all of the reported experiments in this paper along with ZL.W., YP.L., WZ.T., and analyzed all of the data reported. JG.L. is in charge of the whole project. D.F. revising the work critically for intellectual content. CH.L. helped edit the manuscript and assisted with the submission. DX.X. completed the mechanical design and took part in some of the experiments. JB.W. is responsible for the conduct of the experiments.

\section{COMPETING INTERESTS}

The authors declare no competing interests.

\section{ADDITIONAL INFORMATION}

Supplementary information The online version contains supplementary material available at https://doi.org/10.1038/s41526-021-00154-8.
Correspondence and requests for materials should be addressed to D.-X.X.

Reprints and permission information is available at http://www.nature.com/reprints

Publisher's note Springer Nature remains neutral with regard to jurisdictional claims in published maps and institutional affiliations.

\section{cc) (i)}

Open Access This article is licensed under a Creative Commons Attribution 4.0 International License, which permits use, sharing, adaptation, distribution and reproduction in any medium or format, as long as you give appropriate credit to the original author(s) and the source, provide a link to the Creative Commons license, and indicate if changes were made. The images or other third party material in this article are included in the article's Creative Commons license, unless indicated otherwise in a credit line to the material. If material is not included in the article's Creative Commons license and your intended use is not permitted by statutory regulation or exceeds the permitted use, you will need to obtain permission directly from the copyright holder. To view a copy of this license, visit http://creativecommons. org/licenses/by/4.0/.

(c) The Author(s) 2021 